\title{
Folic acid reduces stroke risk
}

Several randomized trials have shown no beneficial effect of folic acid in preventing cardiovascular disease, but some reports have suggested that folic acid supplementation might decrease the incidence of stroke. In a new study published in the Journal of the American Medical Association, the combination of enalapril and folic acid was reported to reduce the incidence of first stroke when compared with enalapril alone.

To assess the efficacy of folic acid in primary prevention of stroke, Huo et al. randomly allocated 20,702 patients with hypertension and no history of stroke or myocardial infarction (mean age 60 years) to receive a single pill of $10 \mathrm{mg}$ enalapril with or without $0.8 \mathrm{mg}$ folic acid for a median of 4.5 years.

Compared with patients who received enalapril alone, those who received the combination therapy had lower risk of first stroke (2.7\% vs 3.4\%; HR 0.79, 95\% CI 0.68-0.93), first ischaemic stroke (2.2\% vs $2.8 \%$; HR $0.76,95 \%$ CI $0.64-$ 0.91 ), and of a composite of cardiovascular death, myocardial infarction, and stroke (3.1\% vs 3.9\%; HR 0.80, 95\% CI 0.69-0.92). An analysis of data stratified according to a polymorphism in the MTHFR gene (C677T), which encodes for the main enzyme in folate metabolism, demonstrated that the patient's baseline folic acid level is an important determinant of risk of stroke and of efficacy of folate therapy.

The mechanisms underlying the interaction between the MTHFR C677T genotype and folic acid level remain to be investigated. However, the findings of this study support folic acid supplementation as a strategy to prevent first stroke in patients with hypertension without a history of myocardial infarction or stroke, particularly in those with a low baseline folate level.

João H. Duarte

Original article Huo, Y. et al. Efficacy of folic acid therapy in primary prevention of stroke among adults with hypertension in China. The CSPPT randomized clinical trial. JAMA doi:10.1001/jama.2015.2274 